\title{
GEOREFERENCIACIÓN DE DATOS OBTENIDOS A TRAVÉS DE RADIO ECO-SONDAJE AL NORTE DE LA PENÍNSULA ANTÁRTICA
}

\author{
GEOREFERENCING DATA OBTAINED THROUGH RADIO ECHO- \\ SOUNDING AT NORTH OF THE ANTARCTIC PENINSULA
}

Carlos Cárdenas ${ }^{1,3}$, Rubén Carvallo ${ }^{1,2}$, Cristian Dodman ${ }^{1}$, Erling Johnson ${ }^{1} \&$ Jorge Hernández $^{1}$

\begin{abstract}
This investigation presents data from working with Global Positioning System (GPS) to complement the work of Radio Echo Sounding from January 2009 at the area next to the Chilean Base Station Bernardo O'Higgins and Jakenau Mountain at north of the Antarctic peninsula (63 $19^{\prime} \mathrm{S}, 57^{\circ} 51^{\prime} \mathrm{W}$ ). The main objective was to georeference the information onto crevasses zones identified by Radio Eco Sounding, using two topographic GPS equipment. The measured points correspond to a grid draw on the area that is normally used for Twin Otter aircraft landing thus, surface and subglacier topography.
\end{abstract}

Key words: Antarctic, GPS, crevasses zones, surface topography.

\section{RESUMEN}

Esta investigación presenta los datos del trabajo realizado con Sistemas de Posicionamiento Global (GPS) como complemento al trabajo de Radio Eco-Sondaje realizado en enero de 2009 en las proximidades a la Estación Base Chilena Bernardo O'Higgins y la montaña Jakenau al norte de la península antártica (63 $13^{\prime}$ S, $\left.57^{\circ} 51^{\prime} \mathrm{W}\right)$. El objetivo principal fue georreferenciar la información correspondiente a la zona de grietas identificada mediante Radio Eco-Sondaje, utilizando dos equipos GPS topográficos. Los puntos medidos corresponden a una grilla cuadricular en la zona que se utiliza habitualmente para el aterrizaje de aviones Twin Otter, habiéndose obtenido la topografía superficial y subglacial.

Palabras clave: Antártica, GPS, zona de grietas, topografía superficial.

1 Dirección de Programas Antárticos, Universidad de Magallanes, casilla 113-D, Punta Arenas, Chile.

2 Departamento de Ingeniería Eléctrica, Universidad de Magallanes, casilla 113-D, Punta Arenas, Chile.

3 Centro de Estudios del Cuaternario, CEQUA, Avenida Bulnes 01855, casilla 113-D, Punta Arenas, Chile. 


\section{INTRODUCCIÓN}

Durante el mes de enero del año 2009, se llevaron a cabo mediciones utilizando equipos GPS topográficos, en las cercanías de la estación base chilena "Bernardo O'Higgins", ubicada en el extremo norte de la Península Antártica, en la costa Sureste del "Estrecho Bransfield" específicamente a los $63^{\circ}$ $19^{\prime} \mathrm{S}$, y $57^{\circ} 52^{\prime} \mathrm{W}$, sobre el domo glaciar donde existe una pista de anevizaje.

Estas mediciones tienen como objetivo complementar por medio de la georreferenciación los trabajos que fueron realizados por un sistema de radar superficial, el cual buscaba dimensionar y analizar la información correspondiente a la zona de grietas identificada en las inmediaciones de la base antes mencionada.

Debido, a las actividades humanas desarrolladas en el área, la presencia de grietas es sumamente relevante, en particular para el tránsito continuo que existe entre la estación base y la pista de anevizaje. Dicha razón fue un argumento más que válido para investigar y conocer la ubicación del campo de grietas de modo de mejorar las condiciones de seguridad de quienes permanecen en la base y deben transitar constantemente por dicho tramo.
Sumado a esto, se debían medir 14 estaciones (balizas) que fueron instaladas y georreferenciadas en las inmediaciones de la pista de anevizaje durante la temporada anterior (enero 2008). El objetivo de la instalación de las balizas es obtener la dirección, velocidad superficial del hielo glaciar para determinar su dinámica, estos parámetros se obtienen mediante la medición de las coordenadas geográficas de cada baliza a través de mediciones con GPS cuya remedición en un periodo determinado permite establecer el desplazamiento da cada una de las balizas. En general la importancia de conocer la dinámica glaciar en la península Antártica obedece al último informe obtenido en la reunión del IPCC del año 2007 en el cual se establecen que uno de los sectores donde incrementó significativamente la temperatura a nivel global es en este sector.

\section{ÁREA DE TRABAJO}

El área donde se realizaron los trabajos se encuentra en las inmediaciones de la estación base chilena "Bernardo O'Higgins", la cual se encuentra ubicada en el extremo norte de la Península Antártica, específicamente en el islote Isabel Riquelme, cuyas coordenadas geográficas son $63^{\circ} 19^{\prime} 15^{\prime \prime} \mathrm{S}$

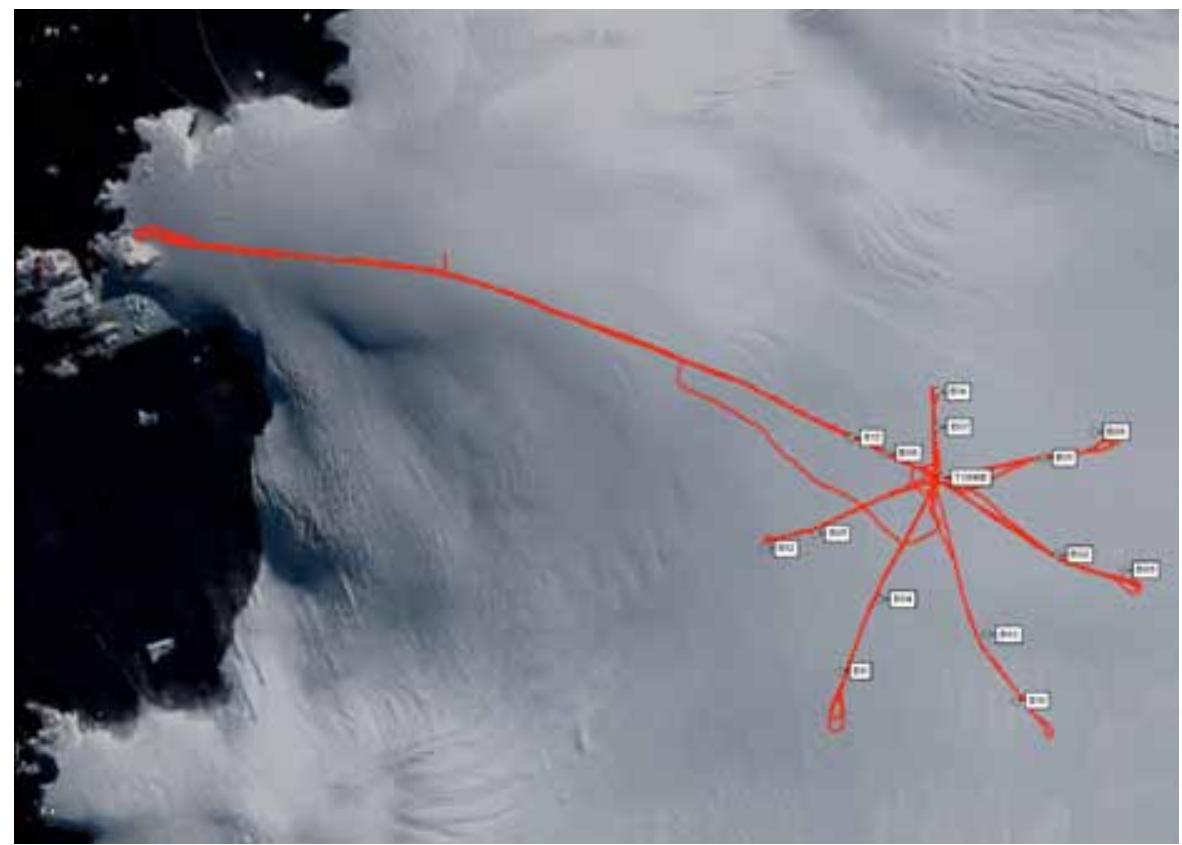

Fig. 1. Área de trabajo en terreno y Posición de las balizas instaladas durante la temporada 2008, en disposición radial en torno a la pista de anevizaje de la estación base chilena "Bernardo O'Higgins". 
y $57^{\circ} 53^{\prime} 55^{\prime \prime} \mathrm{W}$, teniendo una altitud promedio de $12 \mathrm{~m}$. El área donde se trabajo es conocida como la "Meseta de la Infantería", y corresponde al lugar donde se encuentra ubicada la pista de anevizaje correspondiente a la estación base antes mencionada, distante a unos $3 \mathrm{Km}$. de esta, tal como lo muestra la Fig. 1.

\section{MATERIALES Y MÉTODOS}

Las mediciones fueron realizadas haciendo utilización de dos equipos GPS marca NOVATEL, modelo Smart Antenna de alta precisión en frecuencia L1. El primero de estos equipos fue utilizado como estación base (GPS estático), funcionando en modo estático en las inmediaciones del Refugio Rancagua distante a unos $300 \mathrm{~m}$ de la base en cuestión (2432433 Este; 5676138 Norte), mientras que el segundo lo hizo como estación móvil (GPS móvil), funcionando en modo cinemático sobre el mototobogán que transportaba el sistema de radar. Ambos equipos fueron programados de modo de capturar posiciones simultáneamente a intervalos de 1 segundo, mientras se desarrollaban los trabajos de terreno.
Tanto las posiciones capturadas por el GPS estático, como las del GPS móvil, fueron almacenadas en un data-logger de igual forma como se obtuvieron las posiciones en el equipo móvil. Sumado a esto, durante los recorridos, se utilizaron georeceptores de navegación Garmin eMap.

Las trayectorias empleadas por los equipos, fueron planificadas desde el software OziExplorer, empleándose los parámetros del Sistema Geodésico Global (WGS-84) y la proyección Universal Transversal de Mercator (UTM).

En relación al tema de la balizas, estas correspondían a varas de coligue, de $12 \mathrm{~m}$ de largo cada una. Cada baliza fue instalada en el interior del hielo glaciar mediante el uso de una sonda o taladro a vapor, compuesto de un estanque de aluminio, una manguera de alta presión y una punta de lanza. Cada baliza fue instalada a una profundidad estimada de $9 \mathrm{~m}$.

El objetivo de las balizas, era poder establecer estaciones de control para estimar la velocidad, dirección del flujo superficial y el estado del balance de masa del glaciar en esta área, a través de la remedición de las balizas en diferentes temporadas siendo localizadas por medio del uso de equipos GPS.

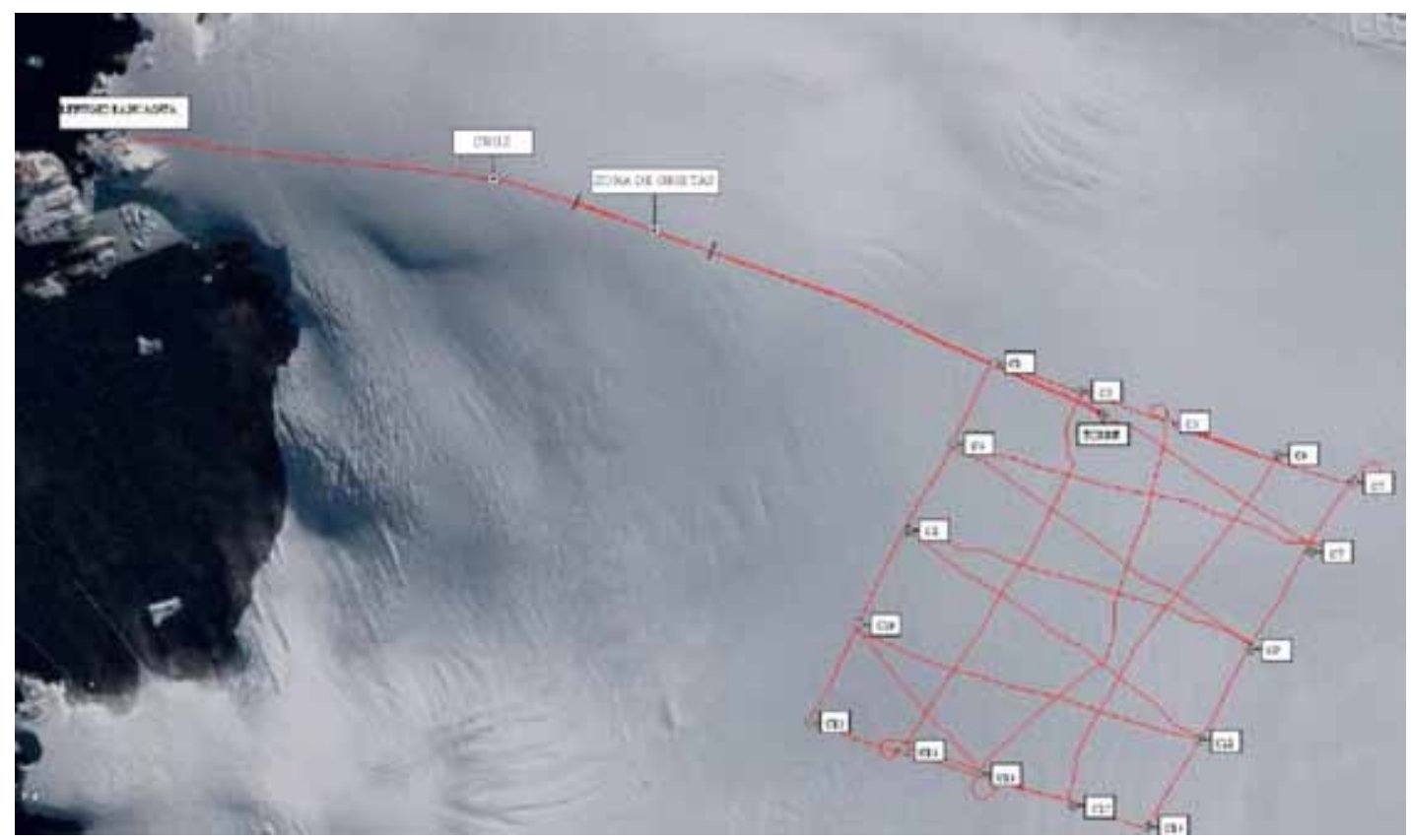

Fig. 2. Tracks y puntos medidos durante la temporada 2009, desde el Refugio Rancagua hasta las inmediaciones de la pista de anevizaje. La grilla está conformada por 16 puntos que va desde $\mathrm{C} 1$ al C16, comenzando al Noroeste del punto TORRE y siguiendo hacia el Este y Sur. 


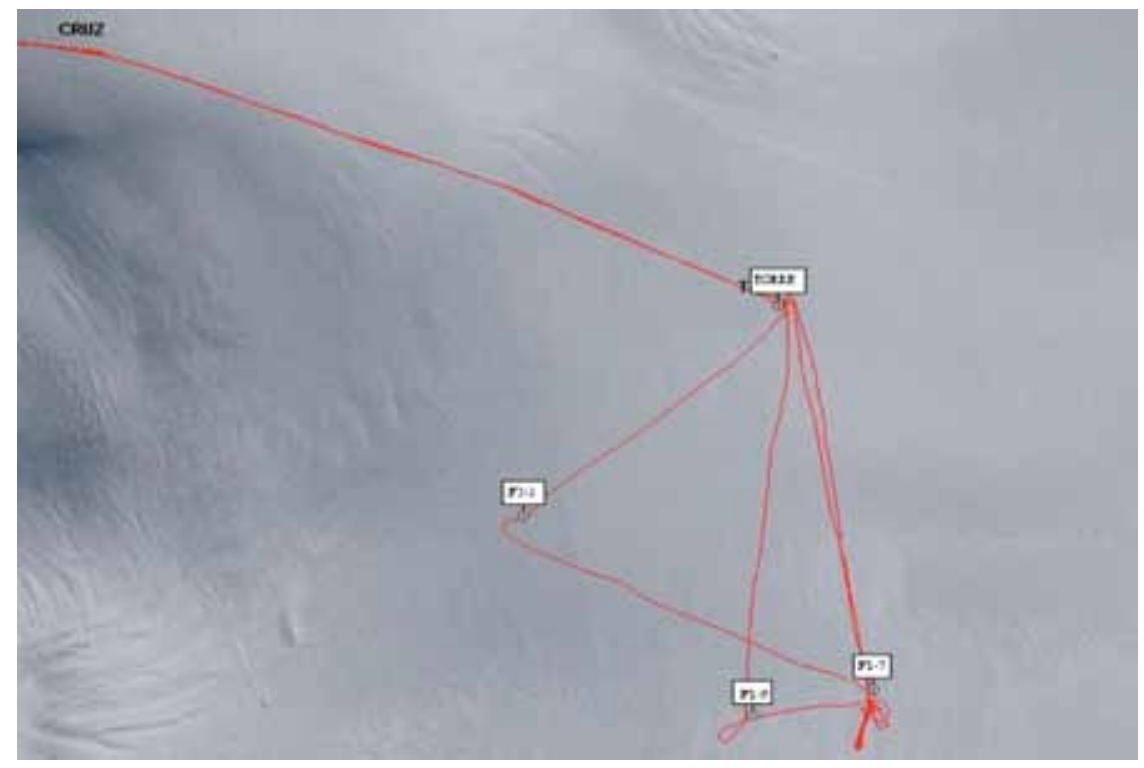

Fig. 3. Trayectos realizados sobre los tres ejes utilizados como pista de anevizaje utilizada normalmente por aviones Twin Otter para realizar abastecimiento logístico a la base Bernardo O'Higgins.

La Fig. 1, muestra la posición en que fueron instaladas las 14 balizas durante la temporada 2008. Estas se posicionaron alrededor de la estructura metálica denominada TORRE en disposición radial, formando dos circunferencias que comenzaron desde el costado derecho de la estructura antes mencionada y considerando 6 ejes de desplazamiento desde el punto central.

\section{RESULTADOS}

Los datos obtenidos por la estación base corresponden a 5 archivos diarios para corrección diferencial, mientras que los obtenidos por la estación móvil al igual que en el caso anterior fueron 5 y correspondían a los recorridos realizados por el sistema de radar superficial.

Se capturaron en total alrededor de $100 \mathrm{Me}$ gabytes de datos de GPS y la evaluación preliminar realizada diariamente una vez finalizada cada faena, mostró que todos fueron de alta calidad.

En primera instancia fue georreferenciado el trayecto de $3 \mathrm{Km}$ que va desde la base O'Higgins hasta la pista de anevizaje, considerando dentro de este tramo el punto denominado CRUZ. Posteriormente, los 16 puntos georreferenciados, así como también la estructura denominada TORRE, conformaron una cuadrícula dibujada en el área de anevizaje (Fig. 2), para finalmente y de modo complementario, georreferenciar y realizar perfiles de radar sobre los tres ejes cuyas direcciones son utilizadas normalmente para anevizar, esto es aproximadamente desde los $170^{\circ}-350^{\circ}, 10^{\circ}-190^{\circ}$ y $50^{\circ}-230^{\circ}$ (Fig. 3).

Dichas mediciones, permitieron determinar la zona de grietas localizada en el tramo que va desde la base a la pista de anevizaje, específicamente radar superficial entre el punto CRUZ y la estación C1, la cual tenia una extensión de aproximadamente $400 \mathrm{~m}$, comenzando unos $200 \mathrm{~m}$ al Este del punto CRUZ. No se encontraron grietas sobre el área de la pista de anevizaje.

A través del uso del equipo GPS y del Sistema de radar fue posible determinar la topografía superficial y subglaciar, más un modelo en tres dimensiones del área que ocupa la pista de anevizaje.

En relación a las balizas instaladas durante la temporada pasada, se visitaron todas las posiciones indicadas basándose en las coordenadas que se tenían, siendo imposible encontrar alguna de ellas, debido principalmente a que la tasa de acumulación de nieve en el periodo 2008-2009, habría sido de 1,9 m aproximadamente, basándonos en la altura del punto TORRE en ambas temporadas. Producto de esto solo fue posible obtener información respecto a este punto, por ello con esta información no fue posible obtener datos de la dinámica glaciar del sector, 
solo pudo determinarse la topografía subglaciar del sector. De acuerdo a lo anterior se espera a futuro realizar una nueva campaña en donde se instalaran nuevamente balizas las cuales serán remedidas en la misma campaña con el objetivo de determinar dirección del flujo glaciar y velocidad.

\section{CONCLUSIONES}

El sistema de radar en complemento con el equipo GPS permitió obtener la información necesaria del área de estudio.

Fue posible, determinar solamente una zona de grietas, localizada en el trayecto entre el punto CRUZ y la estación C1 que se ubica en la zona de la pista de anevizaje y que en cuya área, no se detectaron grietas.

La totalidad de los recorridos fueron registrados con equipos GPS topográficos, en modalidad cinemático, cuyos datos además de permitir conocer exactamente las trayectorias seguidas, sirvieron para elaborar cartografía de la superficie y, en conjunto con los datos de espesor obtenidos a través del sistema de radar, determinar la topografía subglacial.

\section{AGRADECIMIENTOS}

A la Universidad de Magallanes por el importante apoyo financiero brindado bajo el alero del Proyecto Interno N² 27.501 (PR-F1-DPA-2009).
Al Departamento Antártico del Ejército de Chile (DAE), a la Dotación Antártica 2009 de la Base O'Higgins y los miembros de la Brigada de Reparaciones, por el apoyo logístico proporcionado al desarrollo de este proyecto.

A la Armada de Chile y a la Fuerza Aérea de Chile, por cooperar en el traslado de equipos y personal científico a cargo del proyecto respectivamente, desde la ciudad de Punta Arenas hacia el continente Antártico y viceversa.

\section{LITERATURA CITADA}

DLR, 2008. (Deutschen Zentrums für Luft- und Raumfahrt), Satellite image 08 de October de 2008. 08:22:50 UTC, LOW Resolution Quick Looks, TerraSAR-X Spotlight c.

IPCC, 2007. Intergovernmental Panel on Climate Change (IPCC). Fourth Assesment Report: Climate Change 2007.

Paterson, W.S.B. 1981. The physics of glaciers 481, Elsevier Sciences Ltd. pp.

Taurisano, A., S. Tronstadm, O. Brandt \& J. Kohler 2006. On the use of ground penetrating radar for detecting and reducing crevasse-hazard in Dronning Maud Land, Antarctica.

Turner, J. 2004. Review: The El Niño-Southern Oscillation and Antarctica. Internacional Journal of Climatology, 24:1-31 
